\title{
CARTESIAN PRODUCTS WITH INTERVALS
}

M. L. CURTIS ${ }^{1}$

\section{Introduction.}

THEOREM. There exists a combinatorial $n$-manifold $M^{n}$ with boundary $B$ such that $M^{n} \times I=I^{n+1}$ and $\pi_{1}(B) \neq 1$ if and only if $n \geqq 4$.

It has been proved by Bing [1] that such manifolds cannot exist for $n<4$. For $n=4$ an example has been given by Poénaru [6], and we give here a construction which gives such an $M^{n}$ for all $n \geqq 5$. The proof uses the affirmative solution of the generalized Poincaré Conjecture (for $n \geqq 7$ by Stallings [7] and for $n=5,6$ by Zeeman [10]), and the generalized Schoenflies theorem.

\section{Two consequences of the Stallings-Zeeman results. ${ }^{2}$}

Proposition 1. If $M^{n}$ is a contractible combinatorial n-manifold, $n \geqq 5$, and the boundary of $M^{n}$ is $S^{n-1}$, then $M^{n}$ is homeomorphic with $I^{n}$.

Proof. Attach two copies of $M^{n}$ along $S^{n-1}$, using the identity map. The result is a combinatorial $n$-manifold $T^{n}$, and using van Kampen's theorem and duality we get that $T^{n}$ has the homotopy type of $S^{n}$. Since $n \geqq 5, T^{n}$ is homeomorphic with $S^{n}$, and we note that $S^{n-1}$ is nicely embedded. By the Schoenflies theorem ([4] or [2]), it follows that $M^{n}$ is an $n$-cube.

Proposition 2. If $M^{n}$ is a contractible combinatorial $n$-manifold and $n \geqq 5$, then $M^{n} \times I$ is homeomorphic with $I^{n+1}$.

Proof. Let $B$ be the boundary of $M^{n}$. Then the boundary of $M^{n} \times I$ is $T^{n}=\left(M^{n} \times\{0,1\}\right) \cup(B \times I)$. Again it is routine to check that $T^{n}$ has the homotopy type of $S^{n}$, so we conclude that $T^{n}$ is an $n$-sphere. By Proposition 1 we have that $M^{n} \times I$ is homeomorphic with $I^{n+1}$.

3. Construction. Let $P$ be the 2-polyhedron defined by Newman in [5]. This polyhedron has $\pi_{1}(P) \neq 1$ whereas $H_{1}(P, Z)=0=H_{2}(P, Z)$. For $n \geqq 5$ we embed $P$ in $S^{n}$ as a subcomplex and use Newman's result that $\pi_{1}\left(S^{n}-P\right)=1$. Let $T$ be a regular neighborhood of $P$ with $P$ lying in the interior of $T$ (see [8, p. 293]). Then the boundary $B$ of

Presented to the Society, November 18, 1960; received by the editors October 7, 1960.

1 Research partially supported through Research Grant NSF-G14089.

2 These two propositions have also been noted by J. J. Andrews, Imbedding homotopy cells, to appear. 
$T$ is an $(n-1)$-manifold which is also the boundary of $S^{n}-$ Int. $T$. We define $M^{n}=S^{n}-$ Int. $T$.

Now the natural deformation retraction of $T$ onto $P$ induces a map $\phi: B \rightarrow P$ and it is known that $\phi: \pi_{1}(B) \rightarrow \pi_{1}(P)$ is surjective $[3 ; 5]$. Hence $\pi_{1}(B) \neq 1$, and to complete our construction we need only to show that $M^{n}$ is contractible and apply Proposition 2.

Now $\pi_{1}\left(M^{n}\right)=\pi_{1}\left(S^{n}-P\right)$ since $S^{n}-P$ may be deformation retracted onto $M^{n}$, and it follows that $\pi_{1}\left(M^{n}\right)=1$. Since $T$ has the same homotopy type as $P$, it is homologically trivial. By duality, $M^{n}$ is homologically trivial and hence contractible.

\section{REFERENCES}

1. R. H. Bing, $A$ 3-cell is the only object whose cartesian product with an arc is a 4-cell, Abstract 564-257, Notices Amer. Math. Soc. vol. 7 (1960) p. 68.

2. Morton Brown, A proof of the generalized Schoenfies theorem, Bull. Amer. Math. Soc. vol. 66 (1960) pp. 74-76.

3. M. L. Curtis and R. L. Wilder, The existence of certain types of manifolds, Trans. Math. Soc. vol. 91 (1959) pp. 152-160.

4. Barry Mazur, On embeddings of spheres, Bull. Amer. Math. Soc. vol. 65 (1959) pp. 59-65.

5. M. H. A. Newman, Boundaries of ULC sets in euclidean n-space, Proc. Nat. Acad. Sci. U.S.A. vol. 34 (1948) pp. 193-196.

6. V. Poénaru, La decomposition de l'hypercube en produit topologique, Bull. Soc. Math. France vol. 88 (1960) pp. 113-129.

7. John Stallings, Polyhedral homotopy-spheres, Bull. Amer. Math. Soc. vol. 66 (1960) pp. 485-488.

8. J. H. C. Whitehead, Simplicial spaces, nuclei and m-groups, Proc. London Math. Soc. vol. 45 (1939) pp. 243-327.

9. R. L. Wilder, On free subsets of $E^{n}$, Fund. Math. vol. 21 (1933) pp. 156-167.

10. E. C. Zeeman, The generalized Poincare conjecture, Bull. Amer. Math. Soc. vol. 67 (1961) p. 270.

Florida State University 